\title{
Instilled air promotes lipopolysaccharide-induced acute lung injury
}

\author{
YINGGANG ZOU ${ }^{1,2^{*}}$, CHUNLING DONG $^{3 *}$, MINGZHEN YUAN $^{1}$, GUANGYUAN GAO $^{1}$, \\ SIYI WANG ${ }^{1}$, XIAODING LIU ${ }^{1}$, HUIQIAO HAN ${ }^{1}$ and BO LI ${ }^{1}$ \\ ${ }^{1}$ Department of Human Anatomy, College of Basic Medical Sciences, Jilin University, Changchun, Jilin 130021; \\ Departments of ${ }^{2}$ Obstetrics and Gynecology, and ${ }^{3}$ Respiratory Medicine, Second Hospital, \\ Jilin University, Changchun, Jilin 130041, P.R. China
}

Received September 3, 2013; Accepted January 23, 2014

DOI: $10.3892 /$ etm.2014.1523

\begin{abstract}
Optimization of intratracheal instillation is necessary to establish an ideal animal model of acute lung injury (ALI) in order to further reveal the cellular and molecular pathogenesis of ALI. It is possible that instilling air from a prefilled syringe may promote the delivery of reagents into the alveolar spaces, resulting in different pulmonary responses. In the present study, the influence of instilling air by trans-tracheal intratracheal instillation in a lipopolysaccharide (LPS)-induced mouse model of ALI was investigated. The bronchoalveolar lavage (BAL) fluid biochemical index, BAL fluid differential cell counts, lung wet/dry weight ratio, lung histology and BAL fluid interleukin-8 (IL-8) levels were assessed $24 \mathrm{~h}$ subsequent to intratracheal instillation. Instilled air promoted LPS-induced ALI, as indicated by the severity of acute pulmonary inflammation and increased IL-8 release. In conclusion, this study indicates that instilled air may be used to improve the intratracheal instillation procedure and to establish a more reliable animal model of ALI.
\end{abstract}

\section{Introduction}

Acute lung injury (ALI) and its more severe form, acute respiratory distress syndrome (ARDS), are clinical syndromes of acute hypoxic respiratory failure resulting from a variety of direct and indirect injuries to the parenchyma of the lungs (1-3). The mortality rate in patients with ALI/ARDS is $\sim 40 \%$ due to slow progress in understanding the mechanisms responsible for disease pathogenesis (4-6). Lipopolysaccharide (LPS)

Correspondence to: Dr Bo Li, Department of Human Anatomy, College of Basic Medical Sciences, Jilin University, 126 Xinmin Avenue, Changchun, Jilin 130021, P.R. China

E-mail: libosf@gmail.com

${ }^{*}$ Contributed equally

Key words: acute lung injury, lipopolysaccharide, intratracheal instillation, instilled air induces symptoms in animal models that closely resemble ALI/ARDS in humans, highlighting strategies to explore the pathogenesis of ALI/ARDS (7-9).

A reliable experimental animal model is necessary to elucidate the cellular and molecular pathogenesis of ALI/ARDS (10-12). In a previous study of mouse models of LPS-induced ALI, two methods of intratracheal instillation were compared (13). LPS-induced pulmonary inflammatory responses were more severe following trans-tracheal intratracheal instillation relative to those following trans-oral intratracheal instillation, which may be due to more effective delivery of LPS to the lungs by trans-tracheal intratracheal instillation. Of note, delivery of LPS with air from a prefilled syringe was associated with rapid instillation into the lung when using the modified procedure of trans-tracheal intratracheal instillation. Therefore, it is possible that instilled air may promote delivery of LPS into the alveolar spaces, resulting in different pulmonary responses. Instilling air from a prefilled syringe has been used to investigate the pulmonary toxicity of nanoparticles (14). However, the influence of intratracheal air instillation on experimental animal models of ALI has not been assessed.

In the present study, the influence of trans-tracheal intratracheal air instillation on a mouse model of LPS-induced ALI was investigated. The aim of the study was to reveal the role of intratracheal air instillation in LPS-induced experimental animal models of ALI.

\section{Materials and methods}

Animals. Male C57BL/6 mice ( $\mathrm{n}=75$; weight, $20 \pm 2 \mathrm{~g}$ ) were purchased from the Jilin University Animal Center (Changchun, China). The animals were housed in a room at $22^{\circ} \mathrm{C}$ with a 12 -h light/dark cycle (6:00 a.m.-6:00 p.m. light). Mice were fed standard mouse chow and provided water ad libitum. All animal experiments were approved by the Animal Care Committee of Jilin University.

Intratracheal instillation. The procedure for trans-tracheal intratracheal instillation was modified from a previous experiment (15). Briefly, mice were anesthetized by intraperitoneal injection of $0.1 \mathrm{ml}$ pentobarbital sodium $(50 \mathrm{mg} / \mathrm{kg}$; Sigma, 
St. Louis, MO, USA) and placed in a supine position head-up on a board. The board was tilted at a 50-degree angle. A midline incision was made in the neck to expose the trachea. LPS (Sigma) was dissolved in $0.9 \%$ normal saline (NS) at a concentration of $1 \mathrm{mg} / \mathrm{ml}$. LPS at a dosage of $5 \mathrm{mg} / \mathrm{kg}$ or the same volume of NS was drawn into a sterile plastic catheter (up to a premarked level) through a 29-gauge needle and rapidly instilled into the lung with a 1-ml syringe. Following intratracheal instillation, the mice were placed vertically and rotated for 0.5-1 min to ensure even distribution of the instillation within the lungs.

In vivo experimental protocol. Mice $(\mathrm{n}=75)$ were randomly divided into five groups ( $\mathrm{n}=15 /$ group) as follows: Control, NS, NS plus air, LPS and LPS plus air. Mice in the LPS and NS groups were instilled intratracheally with LPS or NS, respectively, as described in the previous section. In the LPS plus air and NS plus air groups, LPS or NS, respectively, was instilled intratracheally with a $1-\mathrm{ml}$ syringe prefilled with $0.1 \mathrm{ml}$ air. Mice in the control group did not undergo any treatment. All mice were sacrificed $24 \mathrm{~h}$ subsequent to intratracheal instillation of LPS or NS.

Bronchoalveolar lavage (BAL). Mice ( $\mathrm{n}=5$ /group) were exsanguinated and sacrificed by removal of the eyeballs following anesthesia with an intraperitoneal injection of $0.1 \mathrm{ml}$ pentobarbital sodium $(50 \mathrm{mg} / \mathrm{kg})$. Following surgical isolation of the trachea, mice were intubated with a 24-gauge cannula. The lungs were flushed with $0.9 \%$ NS in $0.2-\mathrm{ml}$ increments. Recovery of BAL was identical for all experimental groups and the recovery rate was $87 \pm 2 \%$. The BAL fluid was centrifuged for $5 \mathrm{~min}$ at $300 \mathrm{x} \mathrm{g}$, and the supernatant was analyzed to obtain the biochemical index. Levels of lactate dehydrogenase (LDH), alkaline phosphatase (ALP) and total protein were assessed with commercial reagent kits (Nanjing Jiancheng Bioengineering Institute, Nanjing, China) and interleukin- 8 (IL-8) levels were determined with a commercial, specific ELISA kit (R\&D Systems, Minneapolis, MN, USA), used in accordance with the manufacturer's instructions. The pellet was resuspended in $1 \mathrm{ml}$ phosphate-buffered saline with $1 \%$ bovine serum albumin and $0.1 \%$ sodium azide, and a $10-\mu 1$ aliquot was used to count cells. Cell viability was assessed by trypan blue staining (Sigma). In addition, cytospun cells were prepared for Wright's staining and differential cell counting using a cytocentrifuge (Academy of Military Medical Sciences, Beijing, China).

Lung wet/dry weight ratio. Mice (n=5/group) were exsanguinated and sacrificed by removal of the eyeballs. A median sternotomy was performed, and the lungs of each mouse were excised. The wet weight of the exsanguinated whole lungs was measured with an electronic balance, and the lungs were then oven-dried at $60^{\circ} \mathrm{C}$ for $72 \mathrm{~h}$ prior to the dry weight being recorded. The wet-to-dry weight ratio was then calculated.

Lung histology. Mice ( $\mathrm{n}=5 /$ group) were exsanguinated and sacrificed by removal of the eyeballs. The tracheas of the mice were exposed and cannulated with PE-90 tubing, the chests were opened and the lungs were removed and filled with $10 \%$ buffered formalin at an airway pressure of $20 \mathrm{~cm}$
$\mathrm{H}_{2} \mathrm{O}$ for 30 min. Paraffin embedding was performed with the lungs oriented in a prone position, and 5- $\mu \mathrm{m}$ sections were cut for hematoxylin and eosin staining. A lung injury scoring method was utilized to quantify changes in lung architecture, as evidenced by light microscopy. The following variables were used to assess the degree of microscopic injury: alveolar and interstitial edema, neutrophil infiltration and hemorrhage. Each variable was graded according to the severity of injury: no injury, 0 ; injury to $25 \%$ of the field, 1 ; injury to $50 \%$ of the field, 2; injury to $75 \%$ of the field, 3; and diffuse injury, 4 (16). The samples were analyzed based on a scaled grading system by a pathologist who was blinded to the experimental protocol and the sampling region. Three slides from each lung sample were randomly screened and the mean was taken as the representative value of the sample.

Statistical analysis. Statistical analysis was conducted using the SPSS predictive analytics software 18.0 package (SPSS, Inc., Chicago, IL, USA), and all data are expressed as the mean \pm standard error of the mean. One-way analysis of variance followed by Bonferroni (equal variances) or Dunnett's T3 (heteroscedasticity) post hoc test were performed to determine the statistical significance between indicated groups. $\mathrm{P}<0.05$ was considered to indicate a statistically significant difference.

\section{Results}

BAL fluid biochemical analysis. LDH and ALP activities and total protein concentration in the BAL fluid were used as the indicators of cell injury (Fig. 1 and 2). BAL fluid LDH and ALP activities were generally consistent with the degree of cell injury: Cell injury enhances the permeability of the alveolar-capillary barrier, leading to an increased protein concentration in BAL fluid. No significant differences were observed in BAL fluid LDH and ALP activities and total protein concentrations between the sham-treated (NS plus air and NS) and control groups. BAL fluid LDH and ALP activities and total protein concentrations were significantly increased in the LPS plus air and LPS groups compared with the control group (all $\mathrm{P}<0.05)$. These three indices were significantly increased in the LPS plus air group compared with the LPS group (all $\mathrm{P}<0.05)$. The results indicated that the instilled air aggravated LPS-induced cell injury.

BAL fluid differential cell counting. BAL fluid differential cell counting was used to evaluate the number and types of migrated cells, and to further indicate the category and extent of pulmonary inflammation (Fig. 3). No significant differences were observed in the total cell and neutrophil numbers between the sham-treated (NS plus air and NS) and control groups. The total cell and neutrophil numbers were significantly increased in the LPS plus air and LPS groups compared with the control group (all $\mathrm{P}<0.05$ ). Furthermore, the total cell and neutrophil numbers were significantly increased in the LPS plus air group compared with the LPS group (all $\mathrm{P}<0.05$ ). These results indicated that the instilled air promoted LPS-induced neutrophil infiltration into the lungs.

Lung wet/dry weight ratio. Lung wet/dry weight ratio is used as an indicator of pulmonary edema (Fig. 4). No significant 
A

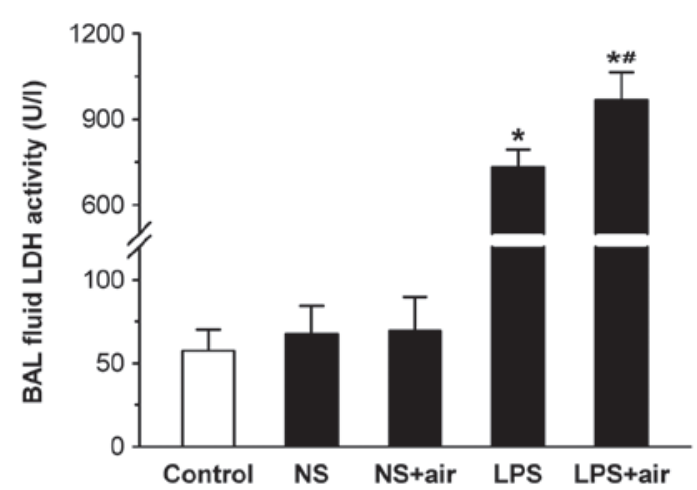

B

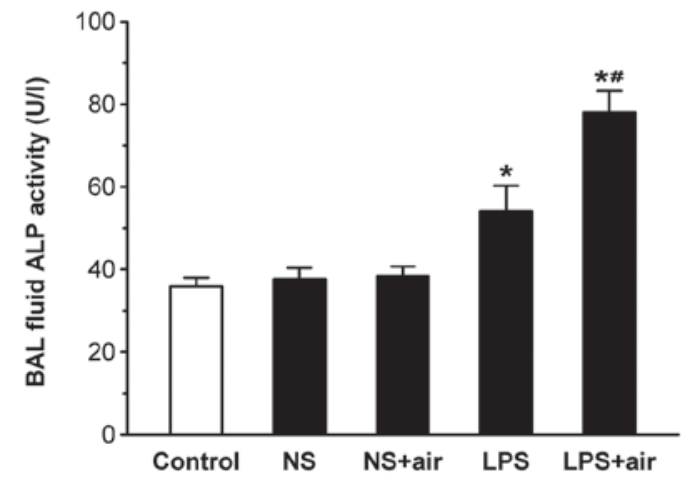

Figure 1. Biochemical evaluation of BAL fluid. (A) LDH and (B) ALP activities. ${ }^{*} \mathrm{P}<0.05$ compared with the control group; ${ }^{~} \mathrm{P}<0.05$ compared with the LPS group. Data are expressed as the mean \pm standard error of the mean $(\mathrm{n}=5)$. BAL, bronchoalveolar lavage; LDH, lactate dehydrogenase; ALP, alkaline phosphatase; NS, normal saline; LPS, lipopolysaccharide.

differences were observed in the lung wet/dry weight ratios between the sham-treated (NS plus air and NS) and control groups. The lung wet/dry weight ratios were significantly increased in the LPS plus air and LPS groups compared with the control group (all $\mathrm{P}<0.05$ ). The lung wet/dry weight ratio was significantly increased in the LPS plus air group compared with the LPS group $(\mathrm{P}<0.05)$. These results indicated that the instilled air exacerbated LPS-induced pulmonary edema.

Lung histology. No marked differences were observed in the lung histology between the sham-treated (NS plus air and NS) and control groups, indicating that intratracheal instillation of NS with or without air did not result in acute lung inflammation (Fig. 5A-C). There were different degrees of fluid accumulation, neutrophil infiltration, congestion and hemorrhage in the LPS plus air and LPS groups compared with the control group. There was more protein-rich fluid, and a greater number of neutrophils and erythrocytes in the alveoli of mice in the LPS plus air group compared with the LPS group (Fig. 5D and E). Edema, neutrophil infiltration and hemorrhage scores were significantly increased in the LPS plus air group compared with the LPS group (all P $<0.05$; Fig. 5F). These results indicated that the instilled air aggravated LPS-induced pathological changes.

BAL fluid IL-8 concentration. BAL fluid IL-8 concentration was assessed due to its important role in the pathogenesis of ALI (Fig. 6). No significant differences were observed in the

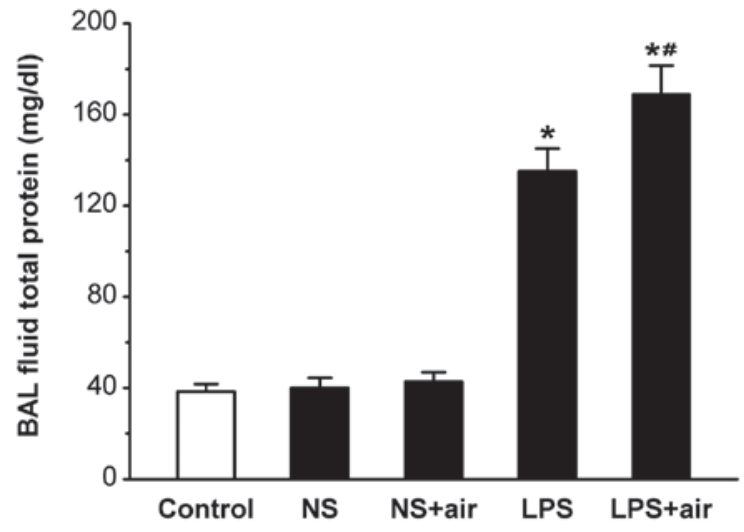

Figure 2. BAL fluid total protein concentrations. ${ }^{*} \mathrm{P}<0.05$ compared with the control group; ${ }^{\text {}} \mathrm{P}<0.05$ compared with the LPS group. Data are expressed as the mean \pm standard error of the mean $(n=5)$. BAL, bronchoalveolar lavage; NS, normal saline; LPS, lipopolysaccharide.

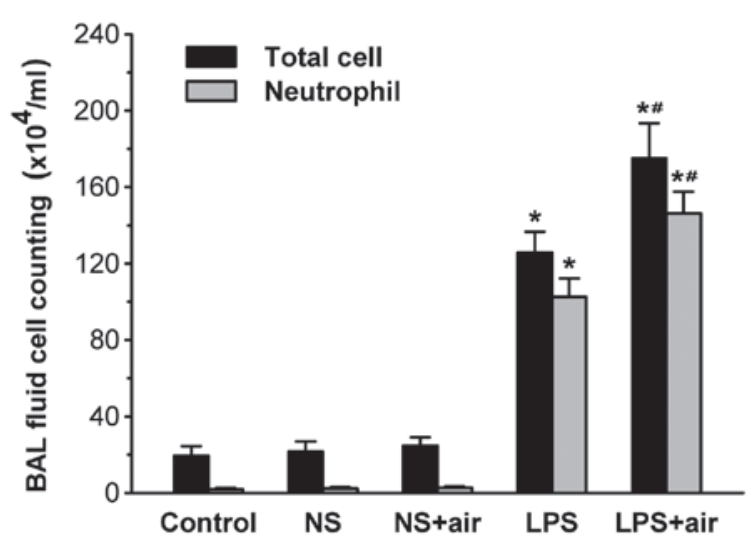

Figure 3. BAL fluid differential cell counting. ${ }^{*} \mathrm{P}<0.05$ compared with the control group; ${ }^{\#} \mathrm{P}<0.05$ compared with the LPS group. Data are expressed as the mean \pm standard error of the mean $(n=5)$. BAL, bronchoalveolar lavage; NS, normal saline; LPS, lipopolysaccharide.

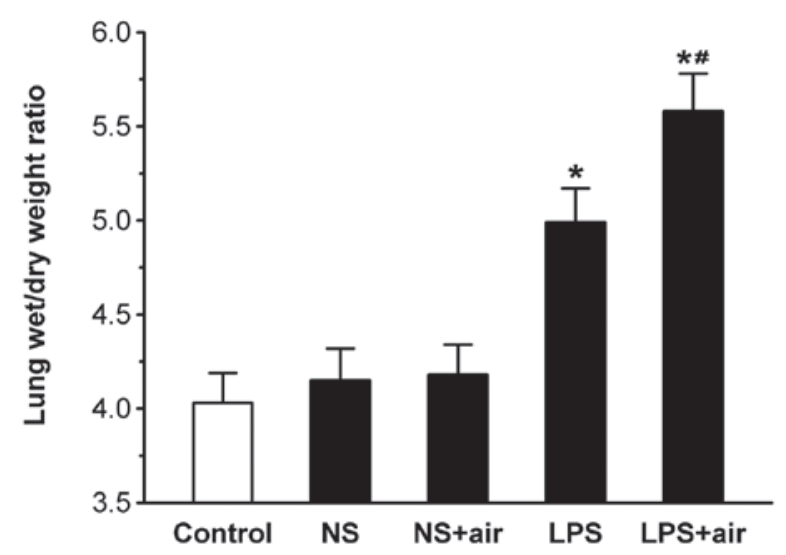

Figure 4. Lung wet/dry weight ratio. ${ }^{*} \mathrm{P}<0.05$ compared with the control group; ${ }^{\#} \mathrm{P}<0.05$ compared with the LPS group. Data are expressed as the mean \pm standard error of the mean $(n=5)$. LPS, lipopolysaccharide; NS, normal saline.

IL-8 levels between the sham-treated (NS plus air and NS) and control groups. Levels of IL-8 were significantly increased in the LPS plus air and LPS groups compared with the control 

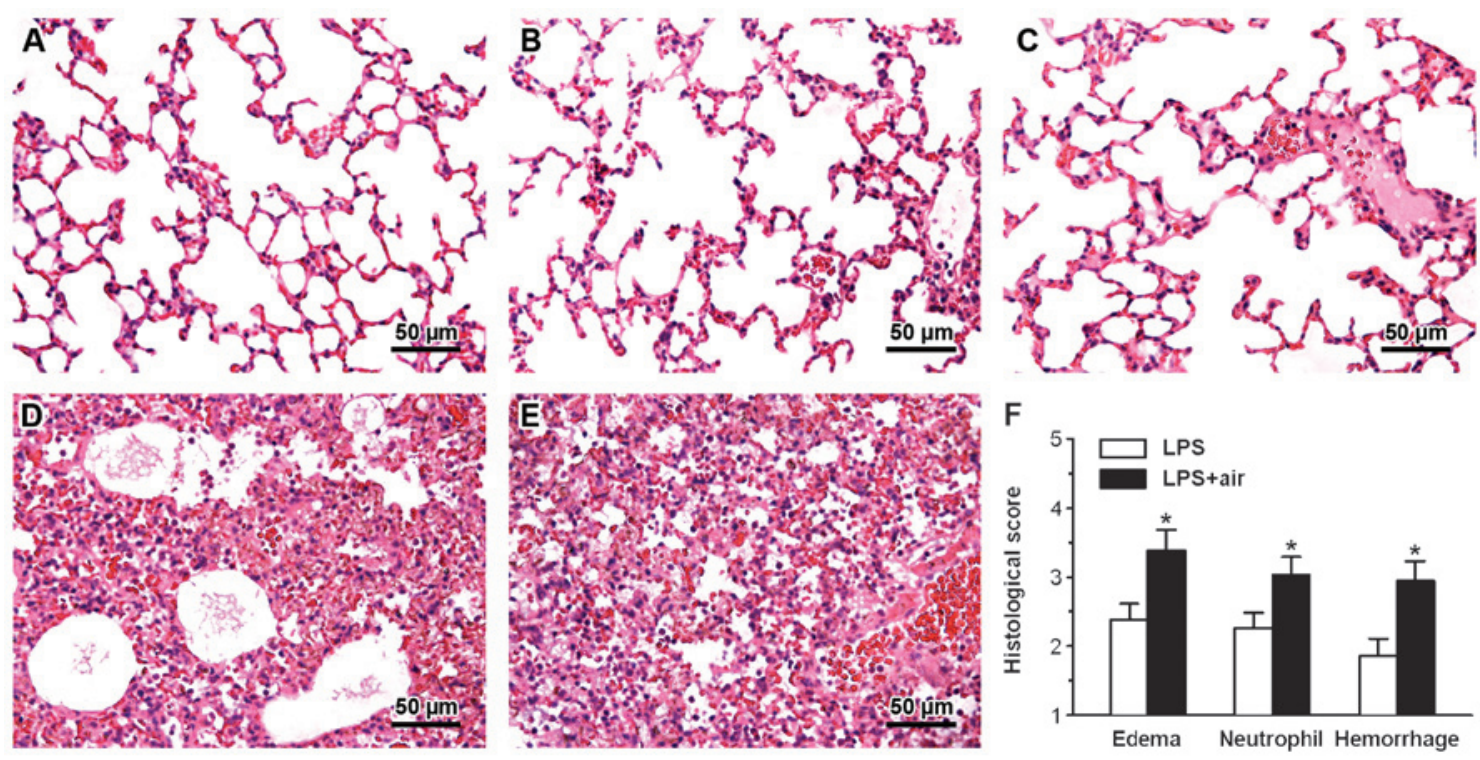

Figure 5. Histological changes in LPS-induced acute lung injury $24 \mathrm{~h}$ subsequent to stimulation (hematoxylin and eosin staining; magnification, $\mathrm{x} 400$; scale bar, $50 \mu \mathrm{m}$ ). (A) Control group; (B) NS group; (C) NS plus air group; (D) LPS group; (E) LPS plus air group; (F) histological score. "P<0.05 compared with the LPS group. Data are expressed as the mean \pm standard error of the mean. LPS, lipopolysaccharide; NS, normal saline.

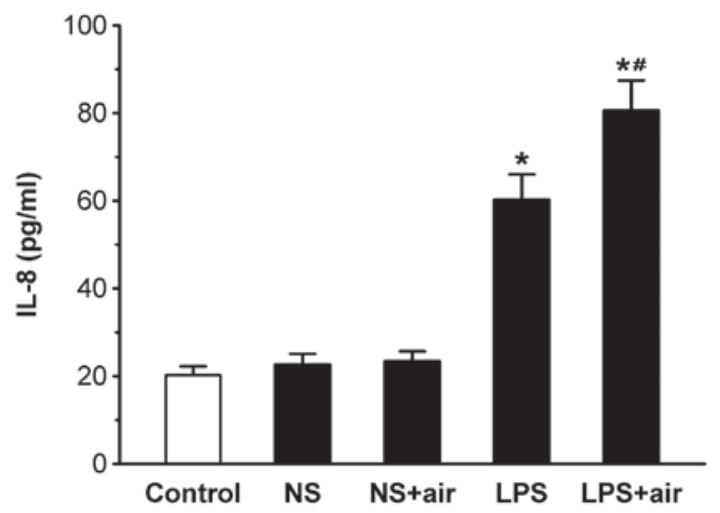

Figure 6. IL-8 release in BAL fluid. * $\mathrm{P}<0.05$ compared with the control group; ${ }^{\text {P }}<0.05$ compared with the LPS group. Data are expressed as the mean \pm standard error of the mean $(n=5)$. IL, interleukin; BAL, bronchoalveolar lavage; LPS, lipopolysaccharide; NS, normal saline.

group (all $\mathrm{P}<0.05)$. IL-8 levels were significantly increased in the LPS plus air group compared with the LPS group $(\mathrm{P}<0.05)$. These results indicated that instilled air increased LPS-induced IL-8 release.

\section{Discussion}

The improvement of intratracheal instillation procedures may facilitate the establishment of an optimal animal model of ALI in order to further reveal the cellular and molecular pathogenesis of ALI. In the present study, the effect of instilling air by trans-tracheal intratracheal instillation on an LPS-induced murine model of ALI was investigated. The results demonstrated that trans-tracheal intratracheal air instillation promoted LPS-induced ALI, as shown by the more severe acute pulmonary inflammation and increased IL-8 release.

Alveolar epithelial and vascular endothelial injury, neutrophil infiltration and increased membrane permeability, leading to pulmonary edema, are involved in the pathogenesis of ALI/ARDS (1). In ALI, binding of LPS to Toll-like receptors on lung cells initiates acute lung inflammation (17). Chemokines are secreted from the stimulated pulmonary epithelium and alveolar macrophages recruit neutrophils into airspaces via the alveolar-capillary barrier (18-20). Activated neutrophils release a variety of mediators, including proteases, reactive oxygen species, histones and peptides, which cause vascular endothelial and alveolar epithelial injuries (21). The subsequent increase in the permeability of the alveolar-capillary barrier leads to the extravascular accumulation of protein-rich edema fluid (22). Disruption of the capacity for fluid clearance and surfactant production due to pulmonary epithelial injury also aggravates pulmonary edema (23). In the present study, instilled air promoted LPS-induced ALI, as evidenced by the more severe acute pulmonary inflammation, including cell injury, neutrophil infiltration and permeability pulmonary edema.

Neutrophils have been indicated to be involved in the pathogenesis of ALI/ARDS, and IL-8 has been identified as the main chemotactic factor for neutrophils in the lung fluid of patients with ALI/ARDS (24-26). IL-8 may enhance the migratory activity of neutrophils and induce migration through the alveolar-capillary barrier, resulting in an accumulation of neutrophils in the alveolar spaces. Therefore, IL-8 levels reflect the severity of ALI in animals and humans. In this study, BAL fluid IL-8 levels were used to further evaluate the influence of instilled air on LPS-induced ALI. Instilled air increased LPS-induced IL-8 release, indicating that instilled air promotes LPS-induced ALI by increasing IL-8 levels. It has been demonstrated that alveolar epithelia are capable of producing a higher level of IL-8 than bronchial epithelia under the stimulation of LPS (13). However, it is possible that the instilled air resulted in delivery of LPS to the alveolar and bronchial epithelial cells in different proportions. Instilled air may drive the discharge of LPS from the syringe, delivering an 
enhanced level of LPS into the alveolar spaces and resulting in greater LPS exposure in the alveolar epithelia, increased IL-8 release and more severe acute pulmonary inflammation.

This study demonstrates that instilled air promotes LPS-induced ALI. To the best of our knowledge, this is the first study to reveal the role of intratracheal air instillation in an LPS-induced experimental animal model of ALI. Instilled air may be used to improve the method of intratracheal instillation and establish a more reliable experimental animal model of ALI. This may enable the further elucidation of the molecular pathogenesis of ALI/ARDS. The instilled air may deliver more LPS into the alveolar spaces, leading to more severe acute pulmonary inflammation. The results of this study may provide guidance for the establishment of other animal models and for approaches to improve drug delivery.

\section{Acknowledgements}

The authors would like to thank Dr Chunling Dong for expert assistance throughout this study. Financial support was provided by the National Natural Science Foundation of China (grant no. 81100030), the Development and Planning Program of Jilin Provincial Science and Technology Department (grant no. 20130522022JH), the Administration of Traditional Chinese Medicine of Jilin Province (grant no. 2012-135), the Jilin University Scientific Frontier and Interdisciplinary Innovative Program (grant no. 450060491515) and Jilin University Innovative Training Program (grant nos. 2012A71198 and 2012C71312).

\section{References}

1. Matthay MA and Zemans RL: The acute respiratory distress syndrome: pathogenesis and treatment. Annu Rev Pathol 6: 147-163, 2011.

2. Ware LB and Matthay MA: The acute respiratory distress syndrome. N Engl J Med 342: 1334-1349, 2000.

3. Li B, Yang J, Huang Q, et al: Biodistribution and pulmonary toxicity of intratracheally instilled graphene oxide in mice. NPG Asia Materials 5: e44, 2013.

4. Spragg RG, Bernard GR, Checkley W, et al: Beyond mortality: future clinical research in acute lung injury. Am J Respir Crit Care Med 181: 1121-1127, 2010.

5. Phua J, Badia JR, Adhikari NK, et al: Has mortality from acute respiratory distress syndrome decreased over time?: A systematic review. Am J Respir Crit Care Med 179: 220-227, 2009.

6. Rubenfeld GD, Caldwell E, Peabody E, et al: Incidence and outcomes of acute lung injury. N Engl J Med 353: 1685-1693, 2005.

7. Muñoz NM, Meliton AY, Meliton LN, Dudek SM and Leff AR: Secretory group V phospholipase A2 regulates acute lung injury and neutrophilic inflammation caused by LPS in mice. Am J Physiol Lung Cell Mol Physiol 296: L879-L887, 2009.

8. Xu XL, Xie QM, Shen YH, et al: Mannose prevents lipopolysaccharide-induced acute lung injury in rats. Inflamm Res 57: 104-110, 2008.
9. Mei SH, McCarter SD, Deng Y, Parker CH, Liles WC and Stewart DJ: Prevention of LPS-induced acute lung injury in mice by mesenchymal stem cells overexpressing angiopoietin 1. PLoS Med 4: e269, 2007.

10. Kim HA, Park JH, Lee S, Choi JS, Rhim T and Lee M: Combined delivery of dexamethasone and plasmid DNA in an animal model of LPS-induced acute lung injury. J Control Release 156: 60-69, 2011.

11. Martin TR and Matute-Bello G: Experimental models and emerging hypotheses for acute lung injury. Crit Care Clin 27: 735-752, 2011.

12. Reiss LK, Uhlig U and Uhlig S: Models and mechanisms of acute lung injury caused by direct insults. Eur J Cell Biol 91: 590-601, 2012.

13. Liu L, Gao Z, Xia C, et al: Comparative study of trans-oral and trans-tracheal intratracheal instillations in a murine model of acute lung injury. Anat Rec (Hoboken) 295: 1513-1519, 2012.

14. Lam CW, James JT, McCluskey R and Hunter RL: Pulmonary toxicity of single-wall carbon nanotubes in mice 7 and 90 days after intratracheal instillation. Toxicol Sci 77: 126-134, 2004.

15. Song YL, Fukuda N, Bai CX, Ma TH, Matthay MA and Verkman AS: Role of aquaporins in alveolar fluid clearance in neonatal and adult lung, and in oedema formation following acute lung injury: studies in transgenic aquaporin null mice. J Physiol 525: 771-779, 2000.

16. Su X, Bai CX, Hong QY, et al: Effect of continuous hemofiltration on hemodynamics, lung inflammation and pulmonary edema in a canine model of acute lung injury. Intensive Care Med 29: 2034-2042, 2003.

17. Matthay MA, Ware LB and Zimmerman GA: The acute respiratory distress syndrome. J Clin Invest 122: 2731-2740, 2012.

18. Li B, Dong C, Wang G, Zheng H, Wang X and Bai C: Pulmonary epithelial CCR3 promotes LPS-induced lung inflammation by mediating release of IL-8. J Cell Physiol 226: 2398-2405, 2011.

19. Bhatia M, Zemans RL and Jeyaseelan S: Role of chemokines in the pathogenesis of acute lung injury. Am J Respir Cell Mol Biol 46: 566-572, 2012.

20. Thorley AJ, Ford PA, Giembycz MA, Goldstraw P, Young A and Tetley TD: Differential regulation of cytokine release and leukocyte migration by lipopolysaccharide-stimulated primary human lung alveolar type II epithelial cells and macrophages. J Immunol 178: 463-473, 2007.

21. Grommes J and Soehnlein O: Contribution of neutrophils to acute lung injury. Mol Med 17: 293-307, 2011.

22. Kropski JA, Fremont RD, Calfee CS and Ware LB: Clara cell protein (cc16), a marker of lung epithelial injury, is decreased in plasma and pulmonary edema fluid from patients with acute lung injury. Chest 135: 1440-1447, 2009.

23. Ware LB and Matthay MA: Alveolar fluid clearance is impaired in the majority of patients with acute lung injury and the acute respiratory distress syndrome. Am J Respir Crit Care Med 163: 1376-1383, 2001.

24. Pallister I, Dent $C$ and Topley N: Increased neutrophil migratory activity after major trauma: a factor in the etiology of acute respiratory distress syndrome? Crit Care Med 30: 1717-1721, 2002.

25. Bao ZY, Ye QW, Gong WH, Xiang Y and Wan HY: Humanized monoclonal antibody against the chemokine CXCL-8 (IL-8) effectively prevents acute lung injury. Int Immunopharmacol 10: 259-263, 2010.

26. Azoulay E, Darmon M, Delclaux C, et al: Deterioration of previous acute lung injury during neutropenia recovery. Crit Care Med 30: 781-786, 2002. 